\title{
NUMERICAL ANALYSIS FOR PRANDTL NUMBER DEPENDENCY ON NATURAL CONVECTION IN AN ENCLOSURE HAVING A VERTICAL THERMAL GRADIENT WITH A SQUARE INSULATOR INSIDE
}

\author{
JAE RYONG LEE ${ }^{1}$ and IL SEOUK PARK ${ }^{2, *}$ \\ ${ }^{1}$ Korea Atomic Energy Research Institute \\ 1045 Daedeok-daero, Yuseong-gu, Daejeon, 305-353, South Korea \\ ${ }^{2}$ School of Mechanical Engineering, Kyungpook National University \\ 1370, Sangyeok-dong, Buk-gu, Daegu, 702-701, South Korea \\ *Corresponding author. E-mail : einstein@knu.ac.kr
}

Received May 16, 2011

Accepted for Publication October 05, 2011

The natural convection in a horizontal enclosure heated from the bottom wall, cooled at the top wall, and having a square adiabatic body in the center is studied. Three different Prandtl numbers $(0.01,0.7$ and 7$)$ are considered for the investigation of the effect of the Prandtl number on natural convection. Adiabatic boundary conditions are employed for the side walls. A twodimensional solution for unsteady natural convection is obtained, using an accurate and efficient Chebyshev spectral methodology for different Rayleigh numbers varying over the range of $10^{3}$ to $10^{6}$. It had been experimentally reported that the heat transfer mode becomes oscillatory when Pr is out of a specific Pr band beyond the critical Ra. In this study, we reproduced this phenomenon numerically. It was found that when $\mathrm{Ra}=10^{6}$, only the case for intermediate $\operatorname{Pr}(=0.7) \operatorname{reached}$ a non-changing steady state and the low and high $\operatorname{Pr}$ number cases $(\mathrm{Pr}=0.01$ and 7) showed a periodically oscillatory fashion hydrodynamically and thermally. The variation of time- and surface-averaged Nusselt numbers on the hot and cold walls for different Rayleigh numbers and Prandtl numbers are presented to show the overall heat transfer characteristics in the system. Further, the isotherms and streamline distributions are presented in detail to compare the physics related to their thermal behavior.

KEYWORDS : Natural Convection, Computational Fluid Dynamics, Spectral Method, Prandtl Number

\section{INTRODUCTION}

Rayleigh-Bénard convection in a horizontal layer of fluid confined between two parallel plates, with the bottom plate heated and the top one cooled, has been studied extensively for over a century. It is well known that the horizontal layer of fluid confined within isothermal walls becomes unstable above a Rayleigh number of 1708 and convective motion sets in the form of steady convective rolls of an aspect ratio (width to height) of about two [1]. With a larger Rayleigh number the flow undergoes a sequence of instabilities and eventually transforms into a turbulent state above a Rayleigh number of about $10^{7}[2,3]$.

For the fundamental purpose of convection heat transfer, i.e. the enhancement of the heat transfer rate, the selection of a working fluid is significant and it should be chosen carefully for its purpose. For example, air is used in most engineering applications due to a convenience of access. Nonetheless, other types of fluid such as distilled water and liquid metal are used in unique environments in closed-loop systems to enhance heat transfer. Many investigations about natural convection with different Prandtl numbers have been carried out for a couple of decades. In geophysical applications, a high Prandtl number case is adequate for the simulation of the motion of Earth's mantle [4]. Nakano et al. [5] performed a numerical simulation of transient natural convection for low Prandtl numbers in a shallow rectangle. Pesso and Piva [6] investigated the influence of the Prandtl number on the average Nusselt number in the laminar regime and showed that the average Nusselt number increases with an increasing Prandtl number, and this is more evident at a high Rayleigh number. The turbulent convective flow was analyzed by Verizicco and Camussi [7] to define the effect of a Prandtl number in a cylindrical cell. Simitev and Busse [8] focused on Prandtl number dependency on a convection-driven flow in a rotating spherical geometry with an electrically conducting fluid in the high Prandtl number region. 
It is important to consider natural convection in complicated geometries in a variety of engineering/ industrial fields, such as the cooling systems of electronic devices, nuclear reactors, and steel-making processes. For example, an accurate understanding of stratified natural convection phenomena is very important in designing and optimizing the heat removal system of a fast sodium reactor. Thus, Choi and Kim [9] analyzed the turbulent natural convection in a shallow enclosure with elliptic blending second-moment closure. A driving mechanism of the energy transport in the passive auxiliary feedwater system (PAFS) of the PWR is another example of the natural convection [10]. The melting pool in the reactor vessel during severe accidents is another application. These phenomena could hardly be a turbulent regime and both experimental and numerical researches were investigated $[11,12]$. Of a variety of geometrical applications, an enclosure with a laterally thermal gradient has been widely studied as a practical example. Vahl Davis [13] numerically analyzed the two-dimensional natural convection in a square enclosure having a lateral thermal gradient. The study was limited to Rayleigh numbers ranging from $10^{3}$ to $10^{6}$ known as a moderate range of Ra. After that, many investigations having an obstacle in the enclosure started to be introduced. Most of them dealt with cases having laterally thermal gradients because of their simplicity, i.e., no oscillating thermal behavior, no change of convective circulating direction and so on. One of the earliest systematic numerical investigations of an enclosure with obstacles was performed by House et al. [14] who considered the influence of a centered conducting body on natural convection within an enclosure. For a given $\mathrm{Ra}$ and $\mathrm{Pr}$, it was found that the case with a thermal conductivity ratio of less than unity (one) enhances the heat transfer. Deng and Tang [15] defined a heat function for the visualization of heat and fluid flow in an air-filled square cavity over a wide range of $\mathrm{Ra}=10^{3}-10^{6}$. Mezrhab et al. [16] considered a radiation-natural convection interaction in a differently-heated cavity with a centered body. Recently, the development of numerical schemes such as an immersed-boundary method makes the simulations more realistic, i.e., more complicated geometry such as an arbitrary location of the square/circular cylinder [17, 18]. Meanwhile, Ha et al. [19] numerically investigated a natural convection in a horizontal enclosure with a vertical thermal gradient at a moderate range of Rayleigh numbers. They analyzed the effect of thermal boundary conditions on a bluff body within the enclosure.

Many investigations have observed the effect of the existence of a bluff body and its thermal boundary condition. However, there is little information about natural convection with vertical thermal gradients for various Prandtl numbers. In this study, the thermal fluid flow and corresponding heat transfer rate with vertical thermal gradients will be considered for various Prandtl numbers. In order to observe the effect of the Prandtl number, we have chosen the values to be $0.01,0.7$ and 7 . Further, a moderate range of Rayleigh numbers $\left(\mathrm{Ra}=10^{3}-10^{6}\right)$ have been applied. In this range, the flow can reach an invariant steady state or a chaotic/periodic oscillating state as a final state according to $\mathrm{Ra}$ and Pr. Even though many practical applications show three-dimensional thermal behavior, this study focuses on a two-dimensional calculation since the range of the Rayleigh number did not show any dominant three-dimensional characteristics. In this study, these indistinct situations depending on $\mathrm{Ra}$ and Pr were successfully simulated and the corresponding thermal fluidic physics are discussed in full by comparing their isotherms, streamlines, the transitions of the average Nusselt number, and so on.

\section{NUMERICAL METHODOLOGY}

The system consists of a horizontal layer of fluid heated from the bottom and cooled from above. The fluid layer has height L, within which a square body with sides of length $\mathrm{W}$, is centered and has an adiabatic thermal boundary condition. The bottom wall is kept at a constant high temperature of $T_{h}$, whereas the top wall of the fluid layer is kept at a constant low temperature of $T_{c}$. The left and right side walls are assumed to be insulated. The geometry is assumed to be invariant along the $\mathrm{z}$ direction and thus the system represents a two-dimensional shape. The fluid properties are also assumed to be constant, except for the fluid density in the buoyancy term, which follows the Boussinesq approximation. The gravitational acceleration acts in the negative $y$-direction. Thus, in the present study, we observe the fluid flow and thermal fields for the natural convection in an enclosure with different Prandtl numbers.

We solve the continuity, Navier-Stokes and energy equations in their non-dimensional forms defined as

$$
\begin{gathered}
\nabla \cdot \mathbf{u}=0 \\
\frac{\partial \mathbf{u}}{\partial t}+\mathbf{u} \cdot \nabla \mathbf{u}=-\nabla p+\operatorname{Pr} \nabla^{2} \mathbf{u}+\operatorname{Ra} \operatorname{Pr} \theta \mathbf{k}_{2} \\
\frac{\partial \theta}{\partial t}+\mathbf{u} \cdot \nabla \theta=\nabla^{2} \theta
\end{gathered}
$$

The dimensionless variables in the above equations are defined as

$$
t=\frac{t^{*} \alpha}{L^{2}}, \quad \mathbf{x}=\frac{\mathbf{x}^{*}}{L}, \quad \mathbf{u}=\frac{\mathbf{u}^{*} L}{\alpha}, \quad P=\frac{P^{*} L^{2}}{\rho \alpha^{2}}, \quad \theta=\frac{T-T_{c}}{T_{h}-T_{c}}
$$

In the above equations, $\rho$ and $T$ represent the density and dimensional temperature, respectively. $\alpha$ is the thermal diffusivity. The superscript ${ }^{*}$ in equation (2) represents the dimensional variables. $\mathbf{u}, p, t$ and $\theta$ are the nondimensional velocity, pressure, time and temperature, respectively. $\mathbf{k}_{2}$ in (1b) is the unit vector normal to the isothermal wall $\left(=\hat{\mathbf{n}} \cdot \delta_{i, 2}, \delta:\right.$ kronecker delta) The above 
non-dimensionalization results in two dimensionless parameters:

$$
\operatorname{Pr}=\frac{v}{\alpha} \text { and } R a=\frac{g \beta L^{3} \Delta T}{v \alpha}
$$

where $v, g$ and $\beta$ are the kinematic viscosity, gravitational acceleration and volume expansion coefficient, respectively. In the simulations reported here the Prandtl number has been taken to be 0.01 corresponding to that of a liquid metal, 0.7 to air and 7 to water.

For the boundary conditions, the velocities are set to zero for all solid walls. The temperature boundary conditions and the conditions at the fluid/body interfaces are as follows:

$$
\begin{array}{ll}
\text { At } \mathrm{y}=0 & \theta=1 \\
\text { At } \mathrm{y}=\mathrm{L} ; & \theta=0 \\
\text { At } \mathrm{y}=0 \text { \& L and fluid/body interface; } & \frac{\partial \theta}{\partial \mathbf{n}}=0
\end{array}
$$

where $\mathbf{n}$ is the vector normal to the boundary. For all simulations, the fluid in the interior is initially assumed to have $\mathrm{T}_{\mathrm{c}}$.

A spectral multi-domain methodology is used for the spatial discretization along the $\mathrm{x}$ and $\mathrm{y}$ directions (Streett and Macaraeg [20]). In this technique, the overall computational domain is subdivided into a number of smaller rectangular subdomains. Within each subdomain a local spectral Chebyshev discretization is defined. Fig. $1 \mathrm{~b}$ shows the computational geometry involving 8 subdomains in the $x-y$ plane, with each subdomain resolved by a $51 \times 51$ grid system. The grid points are the Gauss-Lobatto points corresponding to the Chebyshev expansion within each subdomain and are therefore nonuniformly distributed.

A two-step time-split scheme is used to advance the flow field. First, the velocity is advanced from time level ' $n$ ' to an intermediate level in the two-step time-split procedure by solving the following advection-diffusion equation

$$
\begin{aligned}
\frac{\mathbf{u}_{*}-\mathbf{u}_{n}}{\Delta t}=\frac{\operatorname{Pr}}{2}\left[\nabla^{2} \mathbf{u}_{*}+\nabla^{2} \mathbf{u}_{n}\right] & \\
+ & {\left[\frac{23}{12} \mathbf{N L}_{n}-\frac{16}{12} \mathbf{N L}_{n-1}+\frac{5}{12} \mathbf{N L}_{n-2}\right] }
\end{aligned}
$$

where $\mathbf{u} *$ is the intermediate level velocity and $\mathbf{N L}$ represents the nonlinear buoyancy terms. In the advection-diffusion step, the nonlinear buoyancy terms are treated explicitly using the third-order Adams-Bashforth scheme. The diffusion terms are treated implicitly using the CrankNicholson scheme. Then a Poisson equation for pressure is solved fully implicitly as given below:

$$
\nabla^{2} p_{n+1}=\frac{1}{\Delta t} \nabla \cdot \mathbf{u}_{*}
$$

The final divergence-free velocity field at ' $n+1$ ' is obtained with the following pressure-correction step

$$
\mathbf{u}_{n+1}=\mathbf{u}_{*}-\Delta t \nabla p_{n+1}
$$

The above correction guarantees zero divergence at all the points where the pressure Poisson equation (5b) is satisfied. After obtaining the velocity field, the temperature field is advanced in a similar manner with the third-order Adams-Bashforth scheme for the advection term (denoted below as $\mathrm{AD}$ ) and the Crank-Nicholson scheme for the diffusion term

$$
\begin{aligned}
\frac{\theta_{n+1}-\theta_{n}}{\Delta t}=\left[\nabla^{2} \theta_{n+1}+\nabla^{2} \theta_{n}\right] & \\
+ & {\left[\frac{23}{12} \mathbf{A} \mathbf{D}_{n}-\frac{16}{12} \mathbf{A} \mathbf{D}_{n-1}+\frac{5}{12} \mathbf{A} \mathbf{D}_{n-2}\right] }
\end{aligned}
$$

The above numerical scheme thus requires the solution of a Helmholtz equation for each component of the intermediate velocity (5a), a Poisson equation for pressure

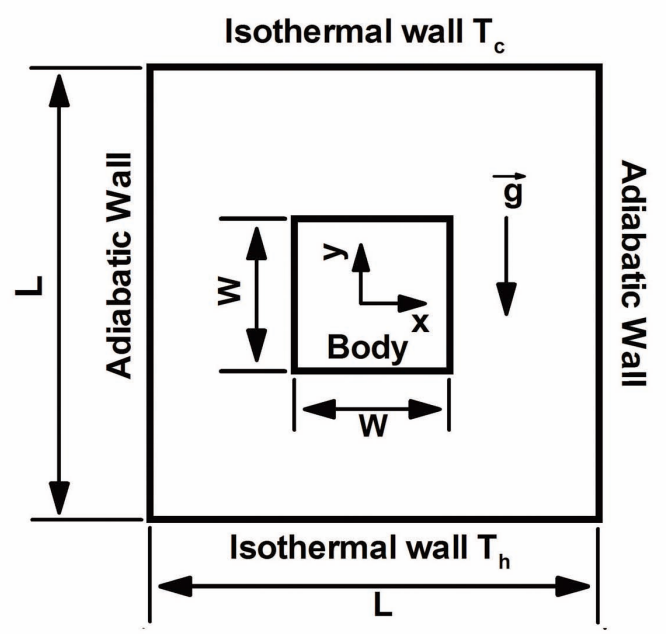

(a) Schematic of the system

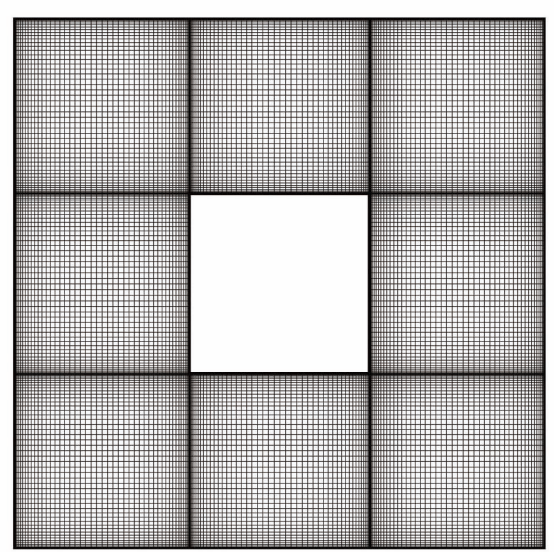

(b) Grid distribution

Fig. 1. Schematic and Grid Distribution of the System. 
(5b) and a Helmholtz equation for the temperature field (5d). In the context of the present spectral multi-domain technique these elliptic equations are solved using a patching technique [21]. The influence matrix technique was also applied to solve the resulting discretized linear system [22].

Once the velocity and temperature fields are obtained, the local, surface-averaged, time-averaged, and time-andsurface-averaged Nusselt numbers are defined as

$$
\begin{aligned}
& N u=\frac{\partial \theta}{\partial \mathbf{n}}, \quad \overline{N u}=\frac{1}{L} \int_{0}^{L} N u d S \\
& \langle N u\rangle=\frac{1}{t_{p}} \int_{0}^{t_{p}} N u d t, \quad\langle\overline{N u}\rangle=\frac{1}{t_{p}} \int_{0}^{t_{p}} \overline{N u} d t
\end{aligned}
$$

where $\mathrm{L}$ is the horizontal extent of the computational domain and $t_{p}$ is the period of time integration. The above quantities are separately computed for the top cold and the bottom hot walls.

The present multi-domain spectral methodology and the computer code used here have been thoroughly validated by comparing the results with those of de Vahl Davis [13], House et al. [14] and Le Quere [23] for a vertical enclosure and with those of Lipps [2] for a horizontal enclosure. For Rayleigh numbers $10^{3}, 10^{4}, 10^{5}$ and $10^{6}$ the benchmark results on the surface-averaged Nusselt number obtained by de Vahl Davis [13] are 1.118, 2.243, 4.519 and 8.800, respectively. The corresponding results obtained with the present code using nine subdomains with $31 \times 31$ grid points resolving each subdomain are respectively, 1.118, 2.246, 4.525 and 8.821 , yielding less than a $0.25 \%$ difference, even for the highest Rayleigh number considered. The problem of natural convection in a vertical enclosure with an interior conducting body studied by House et al. [14] was also considered with the present code. The Nusselt number at the hot wall obtained by House et al. [14] for
$\mathrm{Ra}=10^{5}$ with two different body-to-fluid thermal conductivity ratios of 0.2 and 5.0 are respectively, 4.324 and 4.624 , for a dimensionless body size of $1 / 2$. Again the errors are less than $0.15 \%$. Table 1 shows a summary of these benchmark values. In addition, this code was validated against other physical examples such a turbulent flow in a stirred tank and cross-flow past a circular cylinder with fins $[24,25]$.

\section{RESULTS AND DISCUSSION}

\section{${\underline{\mathrm{Ra}}=\mathbf{1 0}^{3}}^{3}$}

When the Rayleigh number is $10^{3}$, the thermal motion of the fluid reaches a steady state. One can notice that this solution is dominated by conduction rather than convection regardless of the Prandtl number. It is well established that in a pure Rayleigh-Bénard convection in a horizontal layer, the fluid flow becomes unstable above a critical Rayleigh number of about 1708 . Without a body, it is expected that the thermal field shows conduction characteristics exactly and isotherms are orthogonal to the gravitational direction. Fig. 2 shows the isotherms for $\mathrm{Ra}=10^{3}$ and $\operatorname{Pr}=0.7$. Since the other cases for $\operatorname{Pr}$ have quite similar characteristics to the case of $\operatorname{Pr}=0.7$, the isotherms for those cases are omitted in the present paper. The isotherm is not exactly orthogonal to the gravitational direction in the whole domain because of the inner body, and a negligibly small convection stream is detected. Kelly and Pal [26] and Lee et al. [27] noted this kind of appearance which is detected only below the critical Rayleigh number as a quasi-steady conduction state. Despite this nearly non convective heat transfer mode, the surface-averaged Nusselt number is estimated to be 0.77 in this calculation, as depicted in Fig. 13. This is because the spacing between the isotherms is wider because

Table 1. Comparison of the Surface-averaged Nusselt Number Obtained from the Present Calculation with those Obtained from

\begin{tabular}{|c|c|c|c|c|c|c|c|c|}
\hline \multirow[b]{2}{*}{$R a$} & \multirow[b]{2}{*}{$\operatorname{Pr}$} & \multirow[b]{2}{*}{$\Gamma(=W / L)$} & \multirow[b]{2}{*}{$k$} & \multicolumn{4}{|c|}{ Surface-averaged Nusselt Numbers at Hot Wall } & \multirow[b]{2}{*}{ Error $(\%)$} \\
\hline & & & & Present study & $\begin{array}{c}\text { de Vahl } \\
\text { Davis [13] }\end{array}$ & $\begin{array}{c}\text { House et al } \\
{[14]}\end{array}$ & $\begin{array}{l}\text { Le } Q \text { uere } \\
\text { [23] }\end{array}$ & \\
\hline $10^{3}$ & \multirow{7}{*}{0.7} & - & - & 1.118 & 1.118 & - & - & 0 \\
\hline $10^{4}$ & & - & - & 2.246 & 2.243 & - & - & 0.13 \\
\hline \multirow{3}{*}{$10^{5}$} & & - & - & 4.525 & 4.519 & - & - & 0.13 \\
\hline & & 0.5 & 5 & 4.324 & - & 4.324 & - & 0 \\
\hline & & 0.5 & 0.2 & 4.631 & - & 4.624 & - & 0.15 \\
\hline $10^{6}$ & & - & - & 8.821 & 8.800 & - & 8.825 & 0.04 \\
\hline $10^{7}$ & & - & - & 16.524 & - & - & 16.523 & 0.0006 \\
\hline
\end{tabular}
de Vahl Davis [9], House et al. [10] and Le Quere [15]. 
of the existence of an adiabatic body as shown in the isotherms of Fig. 2.

The thermal field in Fig. 2 shows a top-bottom symmetry in the horizontal center plane $(\mathrm{y}=0)$. Bilateral symmetry can also be observed on the vertical plane $(\mathrm{x}=0)$. Note that the governing equations given in Eq. (1) and the boundary conditions of the top and bottom walls should satisfy the following constraints:

Symmetry about $\mathrm{y}=0$ :

$$
\left\{u^{\prime}, v^{\prime}, \theta^{\prime}, x^{\prime}, y^{\prime}\right\} \leftarrow\{u,-v,(1-\theta), x,-y\}
$$

Symmetry about $\mathrm{x}=0$ :

$$
\left\{u^{\prime}, v^{\prime}, \theta^{\prime}, x^{\prime}, y^{\prime}\right\} \leftarrow\{-u, v, \theta,-x, y\}
$$

\section{$\underline{\mathbf{R a}=10^{4}}$}

Fig. 3 shows the surface-averaged Nusselt number at the hot bottom wall at $\mathrm{Ra}=10^{4}$. The flow fields undergo the transient period at the early stage and reach their own final steady states regardless of the Pr. As shown in the magnified graph in Fig. 3, the Prandtl number affects the triggering time to collapse the $\mathrm{x}$ - and $\mathrm{y}$-symmetrical appearance which is shown at $\mathrm{Ra}=10^{3}$. With an increasing Pr, the collapse of the symmetry occurs earlier. Since the momentum diffusion is more dominant than the thermal diffusion as the Pr increases, the convective motion of the fluid in the enclosure is triggered earlier. Thus, the rapid increase of the surface-averaged Nusselt number occurs earlier as Pr increases as shown in Fig. 3(b). On the other hand, for the case of a small Prandtl number $(\operatorname{Pr}=0.01)$, the viscous diffusion is so weak that the convection mode does not appear immediately, but the quasi-steady conduction mode is maintained for a long time. For the cases of Prandtl numbers 0.7 or larger, the surface-averaged Nusselt number oscillates slightly just before reaching a steady state. It seems like a kind of overshooting phenomenon to be settled in an invariant

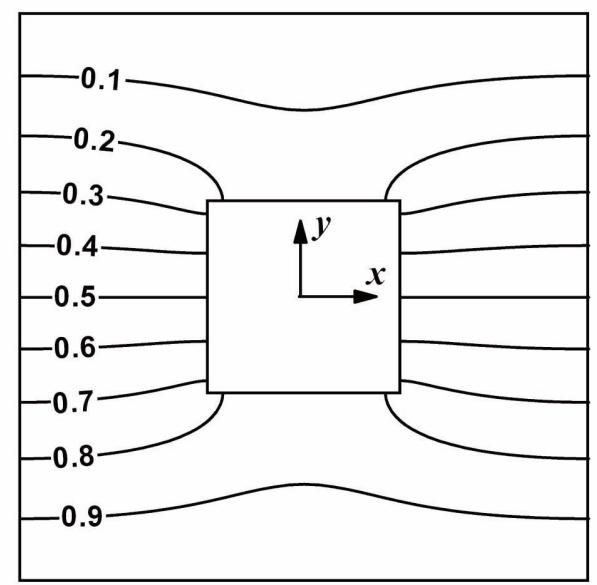

Fig. 2. Distribution of the Isotherms for $\operatorname{Pr}=0.7$ at $\mathrm{Ra}=10^{3}$. steady state. For $\operatorname{Pr}=0.01$, the interaction between the momentum and thermal fields is weak because the thermal fields are fully developed in the conduction mode for a long period in advance. The oscillatory transient (or overshooting) phenomenon before the final steady state is actually expected to be formed by the balancing process between the thermal and hydrodynamic motivations. In cases of extremely small Prandtl numbers, such as $\operatorname{Pr}=0.01$, the thermal fields develop early and stand in the quasi-steady conduction mode as shown in Fig. 3. Thus, when the convective motion is initiated, the oscillatory behavior to balance the effects from both motivating sources does not occur.

To show a detailed scenario about the formation of a circulating roll cell, Fig. 4 represents an isotherm progression for $\operatorname{Pr}=0.7$. The cases for other Prandtl numbers $(\mathrm{Pr}=0.01$ and 7) are not presented in this paper, because they show a very similar developing pattern with only a different triggering time and a difference in the overshooting

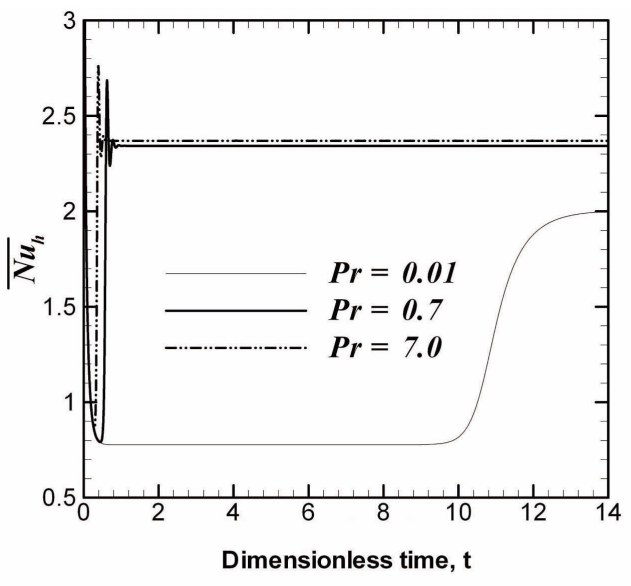

(a) Long-time history

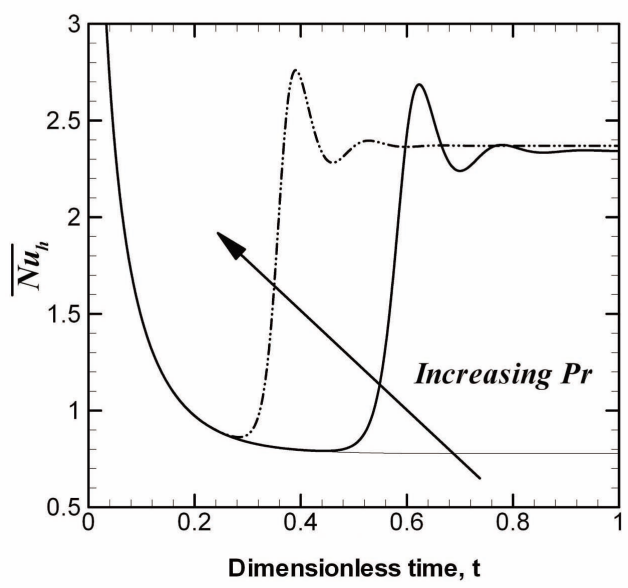

(b) Magnified range

Fig. 3. Surface-averaged Nusselt Number at the Hot Bottom wall at $\mathrm{Ra}=10^{4}$. 
phenomenon before reaching a steady state. At first, the plume from the hot bottom wall rises inside the enclosure (Figs. 4(a)-(d)) and makes the flow fields symmetrical to the $\mathrm{y}$-axis. For $\mathrm{Pr}=0.01$, this quasi-steady state shown in Fig. 4(d) maintains up to $t \approx 10$ due to the weak viscous diffusion. However, the increased buoyant force makes this symmetric field hydrodynamically or thermally unstable. Thus, this symmetric isotherm is broken (Fig. 4(e)) by the circulating flow around the body (Figs. 4(f)-(h)). The circulating direction can be bifurcated either way due to a tiny perturbation or instability. When the flow field reaches its steady state, it shows the diagonal symmetry (Fig. 4(i)).

Diagonal Symmetry:

$$
\left\{u^{\prime}, v^{\prime}, \theta^{\prime}, x^{\prime}, y^{\prime}\right\} \leftarrow\{-u,-v,(1-\theta), L-x,-y\}
$$

Figs. 4(f)-(h) corresponds to the duration of the rapid increase and oscillation for the surface-averaged Nusselt numbers, prior to the final steady state. First, the plumes that originated from the bottom and top walls form the roll cell circulating around the inside body and break the initial symmetrical thermal field in the enclosure. Due to

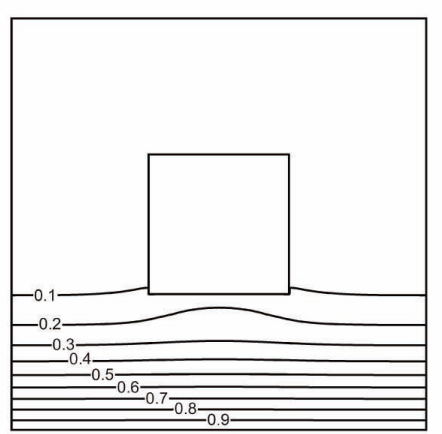

(a) $\mathrm{t}=0.02$

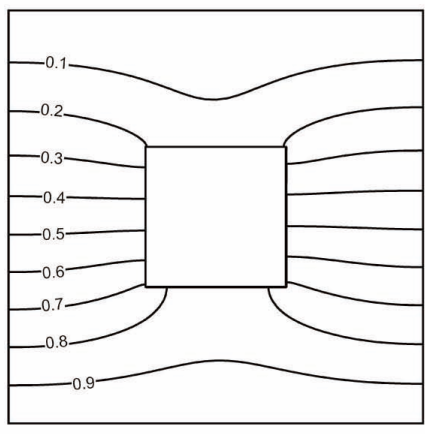

(d) $t=0.23$

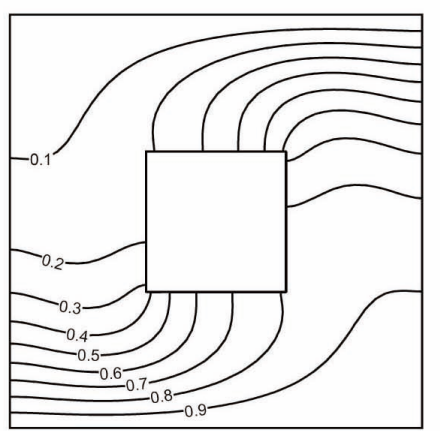

(g) $\mathrm{t}=0.43$

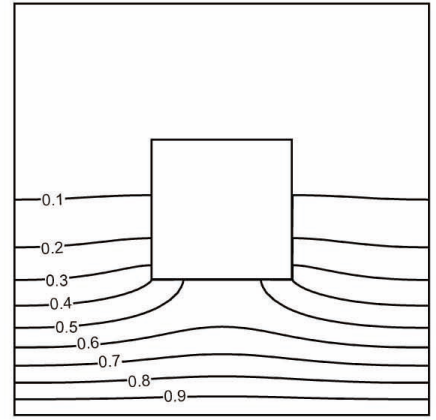

(b) $\mathrm{t}=0.05$

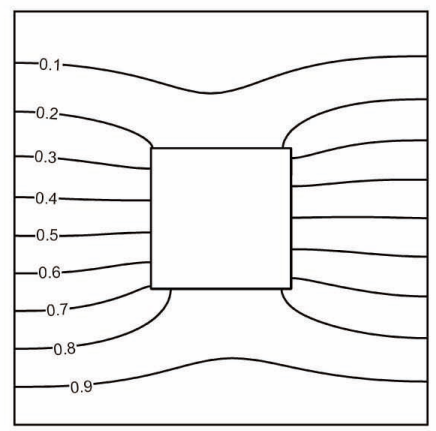

(e) $\mathrm{t}=0.3$

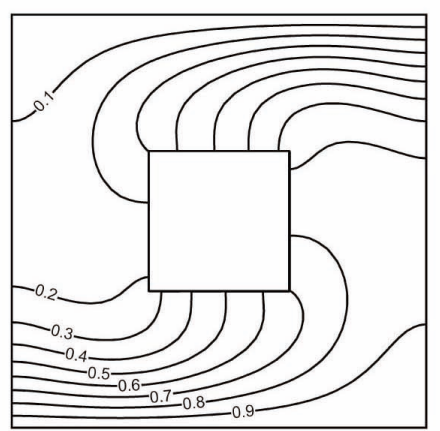

(h) $\mathrm{t}=0.46$

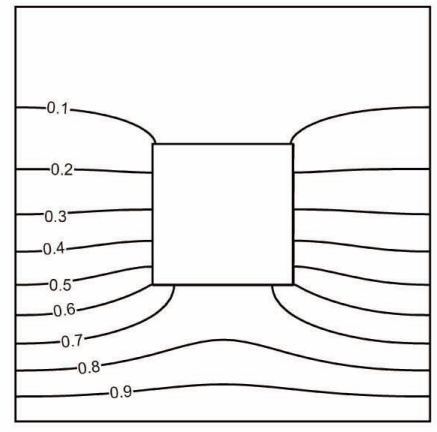

(c) $\mathrm{t}=0.11$

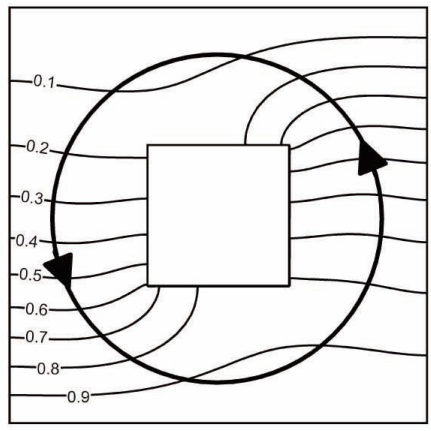

(f) $\mathrm{t}=0.38$

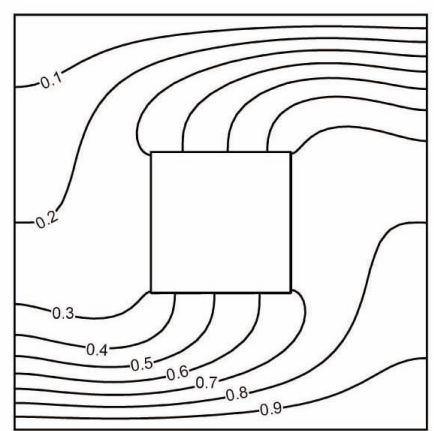

(i) $\mathrm{t}=0.7$

Fig. 4. Snapshots of the Isotherms for $\operatorname{Pr}=0.7$ at $\mathrm{Ra}=10^{4}$. 
the initiation of the convective motion, the heat transfer is boosted on both the top and bottom walls; thus the surfaceaveraged Nusselt number increases rapidly as shown in Fig. 3. A circulating direction of the roll cell can be reversed by any hydrodynamical or thermal instability (called bifurcation phenomena) [28]. In this study, the roll cell circulates in a counterclockwise direction.

Fig. 5 shows the isotherms and streamlines of the roll cells circulating in the enclosure. As is known from the time history of the surface-averaged Nusselt number, the flow patterns for $\operatorname{Pr}=0.7$ and 7 are quite similar. Such a low-Prandtl-number fluid usually has a high Grashof number and a correspondingly high temperature gradient

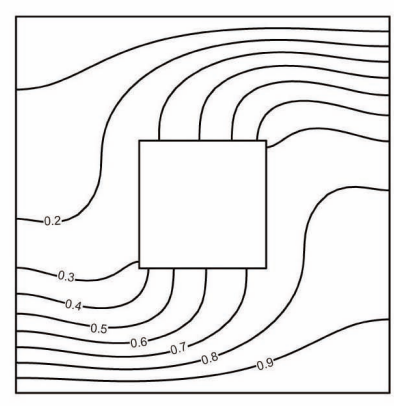

(a-1) Isotherms for $\operatorname{Pr}=0.01$

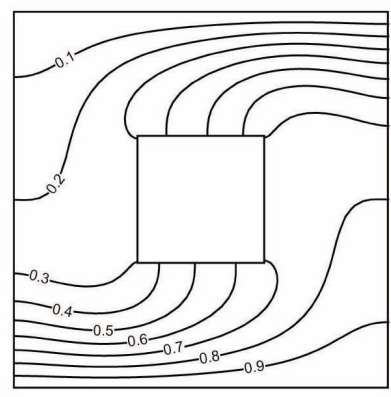

(b-1) Isotherms for $\operatorname{Pr}=0.7$

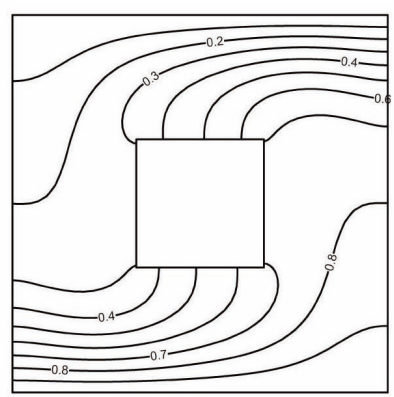

(c-1) Isotherms for $\mathrm{Pr}=7$

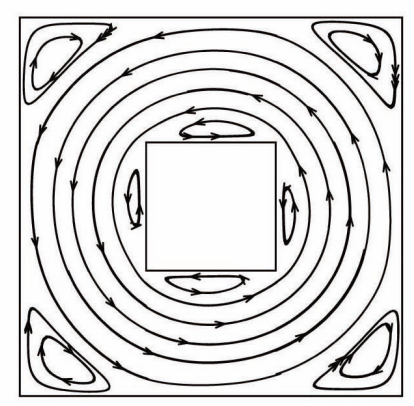

(a-2) Streamlines for $\operatorname{Pr}=0.01$

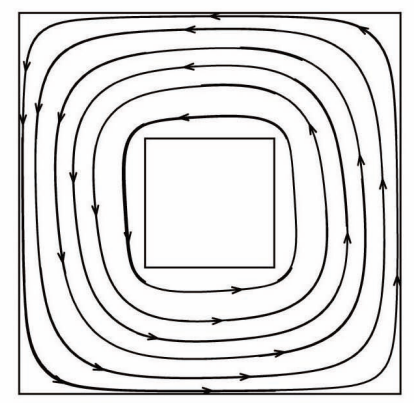

(b-2) Streamlines for $\operatorname{Pr}=0.7$

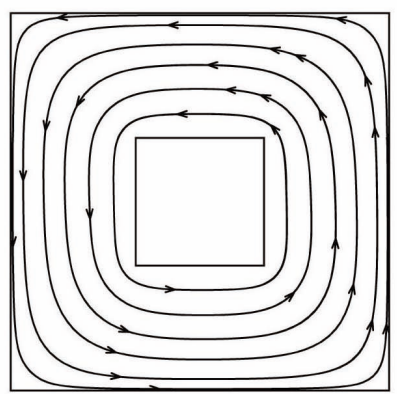

(c-2) Streamlines for $\operatorname{Pr}=7$
Fig. 5. Distribution of the Isotherms and Streamlines at $\mathrm{Ra}=10^{4}$. can occur [29]. From this, the convective motion inside the enclosure becomes more active and produces the secondary vortices detected at each corner of the enclosure and on the surface of the inside body as shown in Fig. 5(a-2). On the other hand, in the cases for high Prandtl numbers, the flow around the body is quite stratified and contacts the top and bottom walls much more widely. The secondary vortices at the corners become very weak and vanish. Thus the resultant surface-averaged Nusselt number at a steady state for a high Pr is larger than in cases of low Prandtl numbers as shown in Fig. 3.

\section{$\underline{\mathbf{R a}=\mathbf{1 0}^{5}}$}

Fig. 6 shows the surface-averaged Nusselt number at the hot bottom wall for $\mathrm{Ra}=10^{5}$. When the Prandtl number is 0.7 or larger, the surface-averaged Nusselt number has an initially oscillatory transient period until it reaches the final steady state, which is similar to that for $\mathrm{Ra}=10^{4}$ with the exception of the initial transient period. Due to the increased buoyant force, the inertial convection of the roll cell around the body makes the flow field more oscillatory. From the magnified figure, it is found that as the $\mathrm{Pr}$

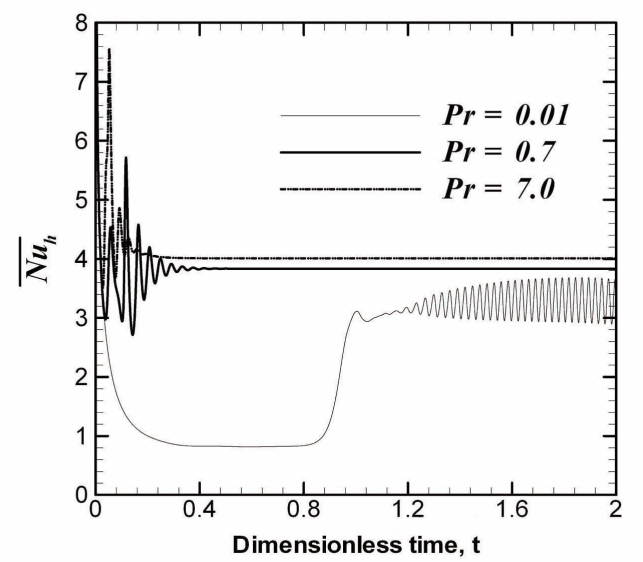

(a) Long-time history

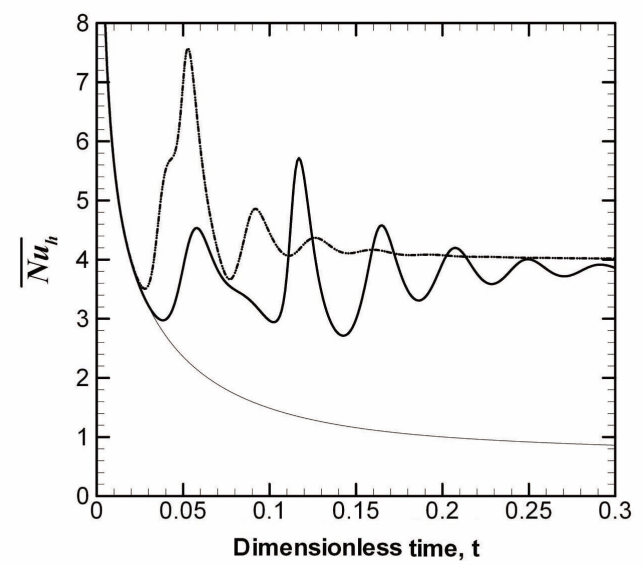

(b) Magnified range

Fig. 6. Surface-averaged Nusselt Number at the Bottom Hot wall at $\mathrm{Ra}=10^{5}$. 
increases, the flow goes to its final steady state earlier because of the larger momentum diffusivity. Unlike those shown in Fig. $4\left(\mathrm{Ra}=10^{4}\right)$, for which the thermal fields satisfy the $\mathrm{x}$ - and $\mathrm{y}$-symmetry prior to break down, the plume starts to move up or down between the side vertical walls and the body. After undergoing the oscillatory duration, the flow fields finally reach a stable state. For $\mathrm{Pr}=0.7$ it takes more time to reach the final steady state. The specific scenario to the final state is well described in Ha et al. [30].

Meanwhile, the flow pattern for $\operatorname{Pr}=0.01$ is different from those for $\operatorname{Pr}=0.7$ and 7 . The flow for $\operatorname{Pr}=0.01$ does not go to the time-invariant steady state but shows oscillatory motion with a high frequency which is not shown in $\mathrm{Pr}=0.7$ or larger. According to previous researchers' findings, one can notice that such a low Prandtl number tends to show a time-dependent flow pattern even though its Rayleigh number slightly exceeds the critical Rayleigh number and the Nusselt number slowly increases [31, 32]. The initial quasi-steady conduction mode is maintained until $\mathrm{t}=0.8$ and then the convective flow begins to circulate around the body. Next, the oscillatory pattern appears and is continuously maintained. This behavior of the surfaceaveraged Nusselt number is quite similar to the results of Arcidiacono et al. [33] who tested a volumetrically heated rectangle.

The roll cell behavior in the oscillatory region is shown in Fig. 7. Since the oscillation of the surface-averaged Nusselt number acts like a sinusoidal wave, Figs. 7(a) to (d) correspond to the maximum/minimum value and inflection points in the interim. At each state, the flow field satisfies the diagonal symmetry. When the surfaceaveraged Nusselt number is a locally high peak as shown in Fig. 7(a), the roll cell's circulation is accelerated and thus the resulting secondary vortices at the four corner points and near the surfaces of the inner body are enlarged. It makes denser isotherms form near the top and bottom walls. During the transition from the high to low peaks of the surface-averaged Nusselt number (Fig. 7(a) to Fig. 7(c)), physics thermally and dynamically interacting between the main roll cell and the secondary vortices appear. As the roll cell circulates continuously, it tends to increase its

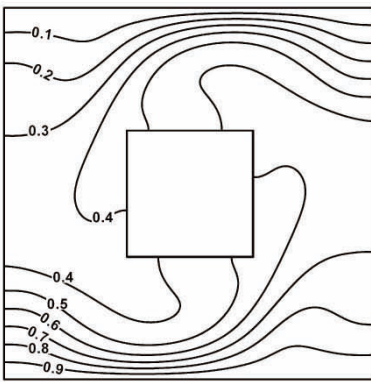

Isotherms

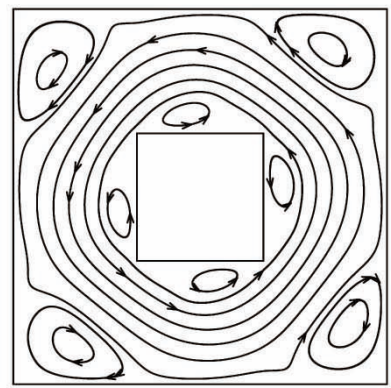

Streamlines

(a) High peak

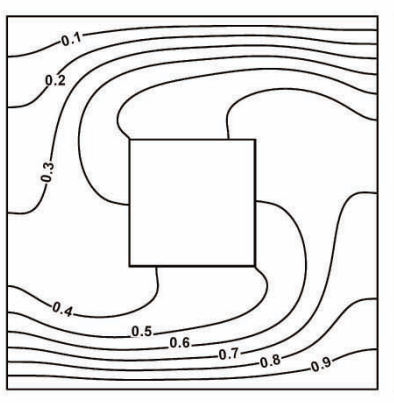

Isotherms

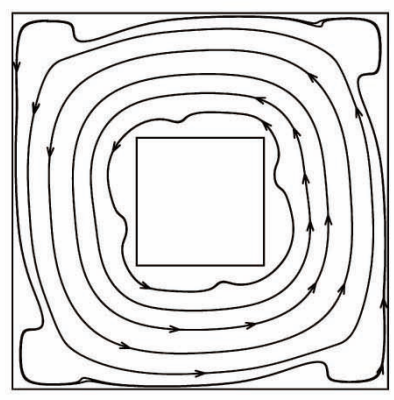

Streamlines

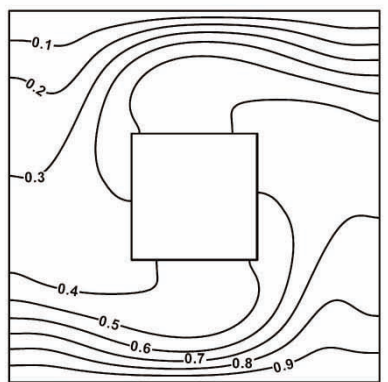

Isotherms

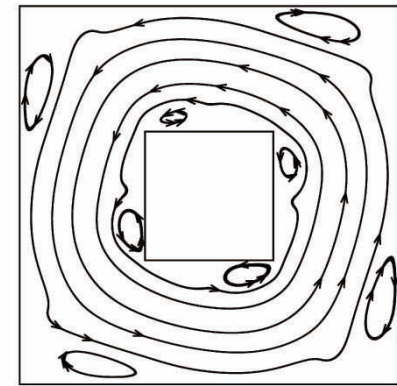

Streamlines

(b) High-to-low

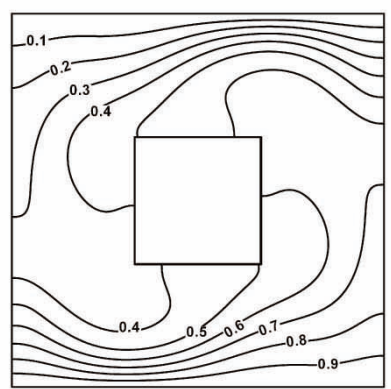

Isotherms

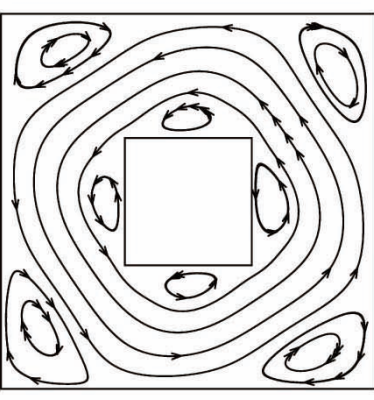

Streamlines

(c) Low peak

(d) Low-to-high

Fig. 7. Snapshots of the Isotherms and stream Lines for $\operatorname{Pr}=0.01$ at $\mathrm{Ra}=10^{5}$. 
size and simultaneously suppresses the secondary vortices. As a result, the secondary vortices change their shapes, i.e., vertically elliptic at the top left and bottom right corners (Fig. 7(a)) to horizontally elliptic at the top right and bottom left (Fig. 7(b)). In addition the main roll cell becomes larger but weaker (lower velocity near the wall surfaces). Fig. 7(c) shows the thermal distribution when the surfaceaveraged Nusselt number is observed to be a low peak. The intensity of the roll cell decreases and the flow is stratified in the interior. Therefore, the heat transfer rate is minimized.
Fig. 8 shows the isotherms and streamlines for different $\mathrm{Pr}$ at $\mathrm{Ra}=10^{5}$. Fig. 8(a) is a long-time averaged one only for the oscillatory range, whereas Figs. 8(b) and (c) is for the final steady state. For $\mathrm{Pr}=0.01$, the time-averaged roll cell consists of the almost circular main roll cell and the pairs of the secondary vortices due to the periodic appearance of the vertically and horizontally elliptic vortices at each corner as shown in Fig. 7. For the cases $(\mathrm{Pr}=0.7$ and 7) having a time-invariant steady solution, the thermal field is more stratified and the sizes of the secondary vortices are smaller or nearly disappear as the Pr increases. This

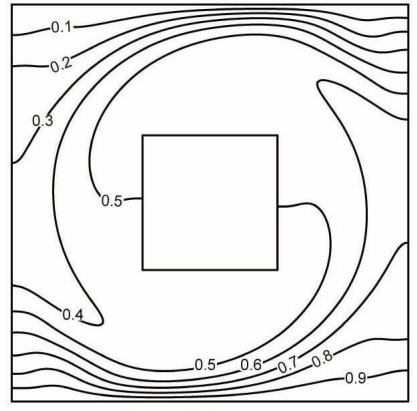

(a-1) Isotherms for $\operatorname{Pr}=0.01$

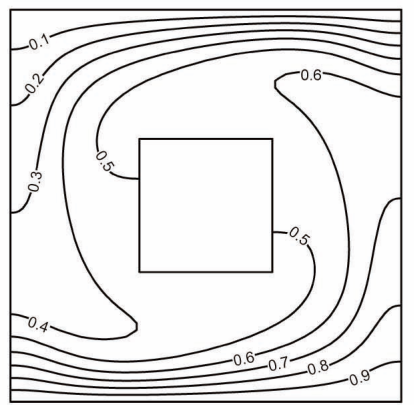

(b-1) Isotherms for $\operatorname{Pr}=0.7$

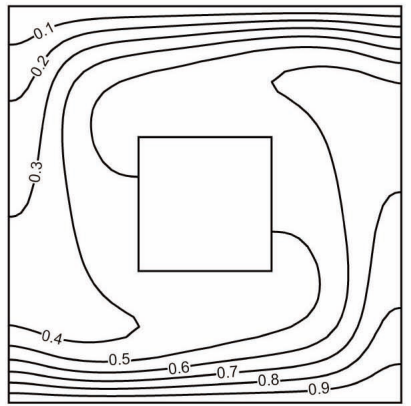

(c-1) Isotherms for Pr=7

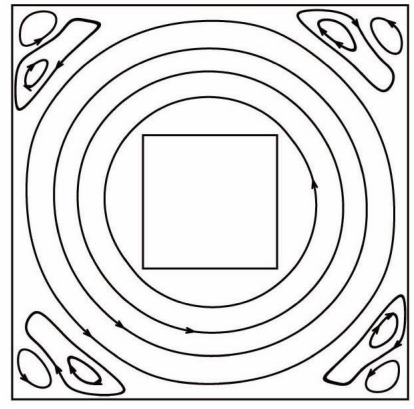

(a-2) Streamlines for $\operatorname{Pr}=0.01$

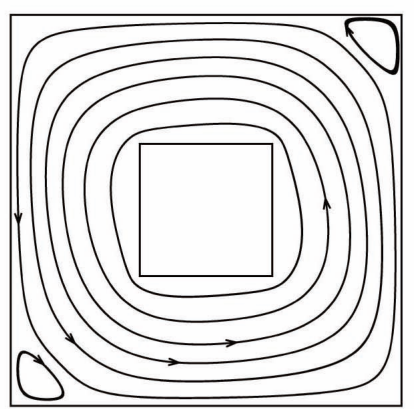

(b-2) Streamlines for $\operatorname{Pr}=0.7$

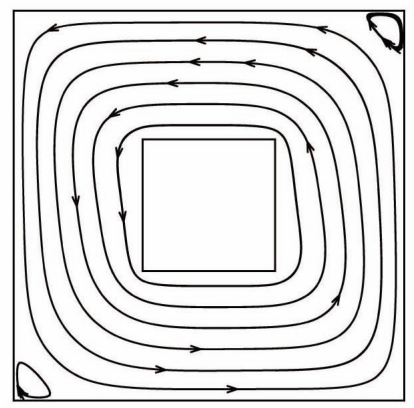

(c-2) Streamlines for $\operatorname{Pr}=7$

Fig. 8. Distribution of the Isotherms and Streamlines at $\mathrm{Ra}=10^{5}$. 
also induces a slightly better heat transfer than the case of $\operatorname{Pr}=0.01$ having relatively larger secondary vortices.

\section{$\underline{\mathbf{R a}}=\mathbf{1 0}^{6}$}

Fig. 9 shows the surface-averaged Nusselt number at the hot wall as a function of time for different Prandtl numbers at $\mathrm{Ra}=10^{6}$ and its power spectrum is shown in Fig. 10. The power spectrum (called the power spectral density function, PSD) shows the strength of the fluctuations as a function of frequency. Computation of the power spectrum is done by computing the autocorrelation function and then transforming it by the Fourier transform. When the Prandtl number is 0.7 , the flow reaches a steady state even though it undergoes an initial oscillatory transient regime, whereas, the cases for $\mathrm{Pr}=0.01$ and 7 do not reach an unchanged final steady state, as shown in Fig. 9.

The flow field for $\operatorname{Pr}=0.01$ oscillates with a single dominant frequency as shown in Fig. 10(a). This thermal behavior of the low-Prandtl-number fluid has already been explained in Ref. [33] and dealt with in the previous explanation for $\mathrm{Ra}=10^{5}$. The final thermal-fluidic behavior, however, for $\mathrm{Pr}=7$ transits from the steady state to a periodically oscillatory pattern (as shown in Fig. 9) at $\mathrm{Ra}=10^{5}$ with couples of dominant frequencies at $\mathrm{Ra}=10^{6}$ (as shown in Fig. 10 (b)). To judge the truth of this nonmonotonic result, that only the case of intermediate Prandtl number $(\mathrm{Pr}=0.7)$ finally goes to the time-invariant steady state, the grid dependency of the solution was verified. The fine grid system up to $123 \times 123$ per unit subdomain (121,032 in total) was tested so that the same phenomenon was observed.

Fig. 11 shows snapshots of the instantaneous isotherms during the oscillation period for $\operatorname{Pr}=7$. Fig. 11(a)-(j) correspond to the time of a-j in Fig. 11(k), respectively. The difference from the case of $\mathrm{Pr}=0.01$ is that a circulating direction of the roll cell is converted periodically as time goes by.

The circulating direction changes every two and half periods as shown in Fig. 11(k). The time period for the clockwise circulation is also shorter than the counterclockwise circulation. However this can be altered according to the

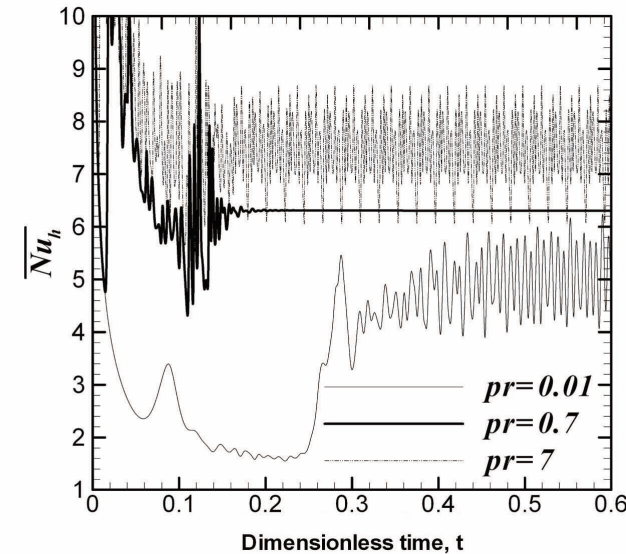

(a) Long-time history

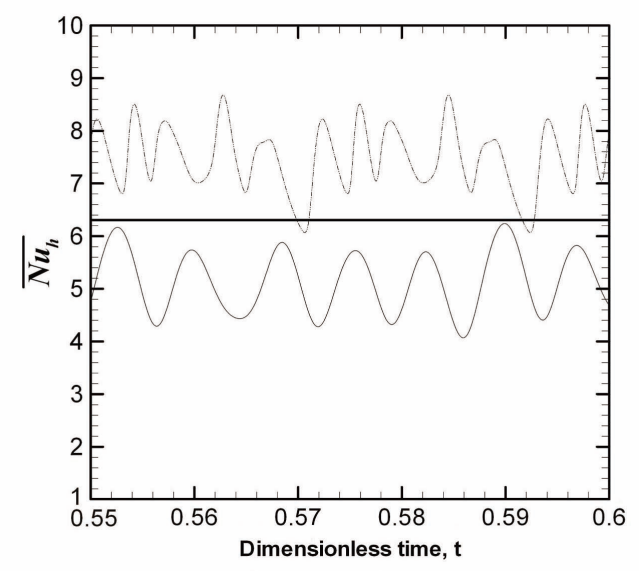

(b) Magnified range

Fig. 9. Surface-averaged Nusselt Number at the Hot Bottom wall at $\mathrm{Ra}=10^{6}$.

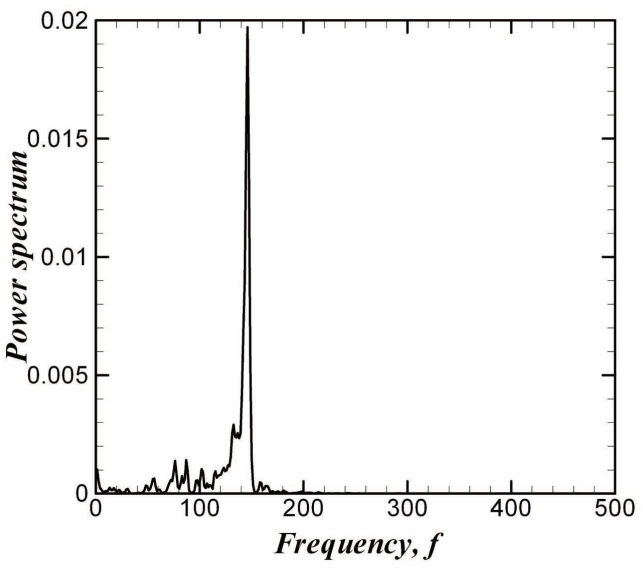

(a) $\operatorname{Pr}=0.01$

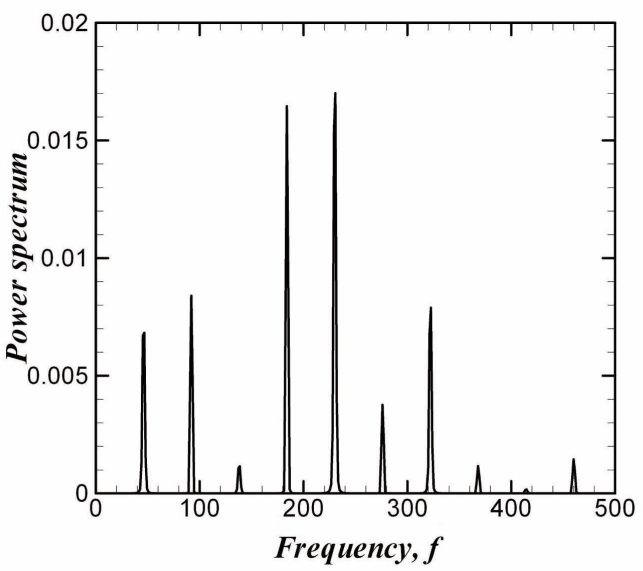

(b) $\operatorname{Pr}=7$

Fig. 10. Power Spectrum of the Surface-averaged Nusselt Number at the Hot wall at $R a=10^{6}$. 
bifurcation theory [28] and coincides with the case of $\operatorname{Pr}=7$, which has several dominant frequencies as shown in Fig. 10(b).
Fig. 12 shows the time-averaged isotherms and streamlines of $\mathrm{Ra}=10^{6}$. The figure for $\mathrm{Pr}=0.7$ is the steady solution. The solutions for $\operatorname{Pr}=0.01$ and 0.7 basically show

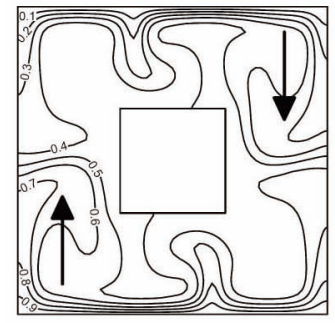

(a) $t=1.015$

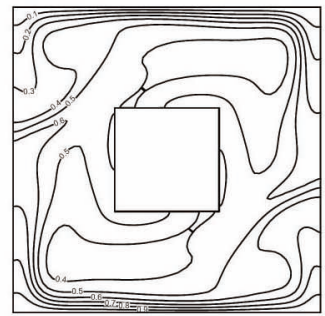

(e) $t=1.022$

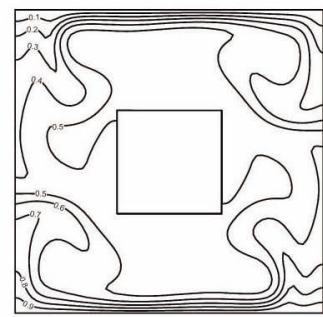

(i) $\mathrm{t}=1.031$

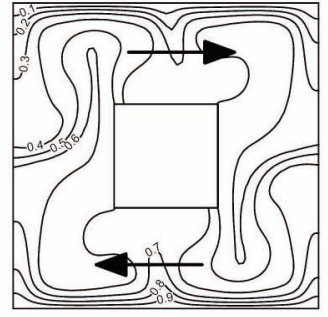

(b) $\mathrm{t}=1.017$

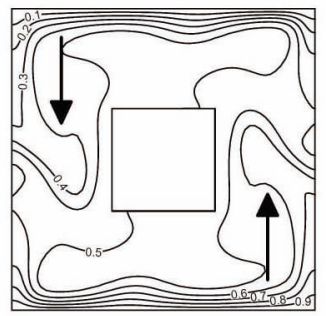

(f) $\mathrm{t}=1.025$

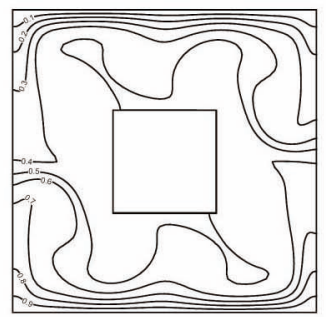

(j) $\mathrm{t}=1.035$

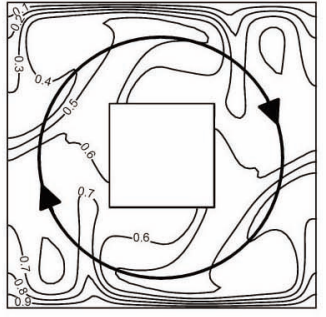

(c) $\mathrm{t}=1.019$

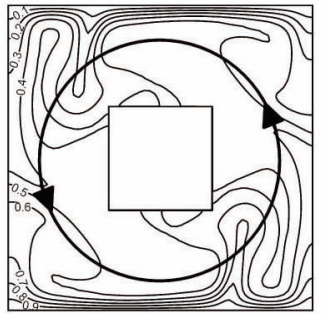

(g) $t=1.027$

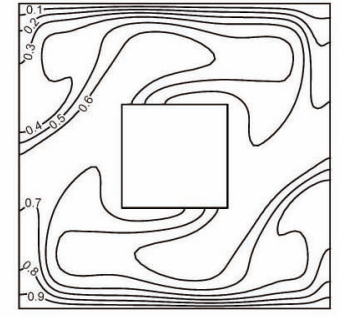

(d) $\mathrm{t}=1.020$

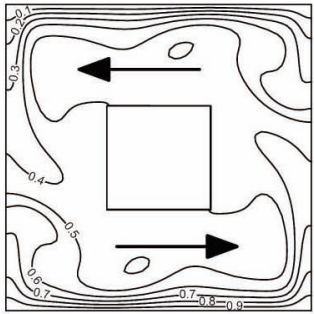

(h) $\mathrm{t}=1.029$

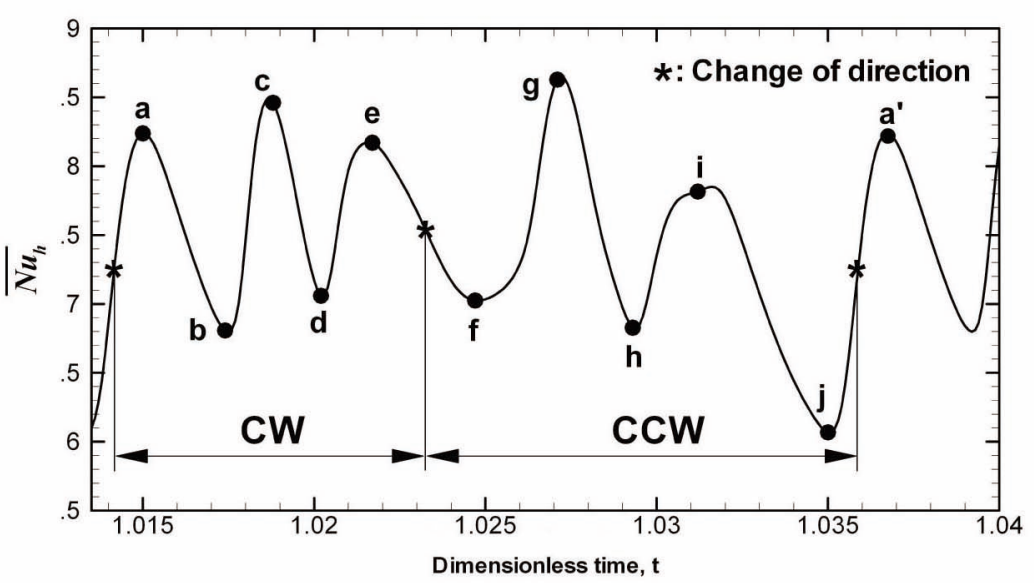

(k) Surface-averaged Nusselt number corresponding to snapshots for $\operatorname{Pr}=7$

Fig. 11. Snapshots of the Isotherms and Periodic Change of Averaged Nusselt Number for $\operatorname{Pr}=7$ at $\operatorname{Ra}=10^{6}$. 


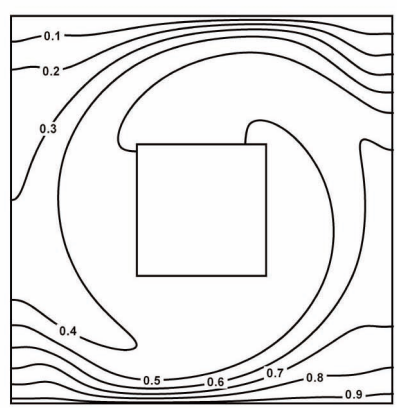

(a-1) Isotherms for $\operatorname{Pr}=0.01$

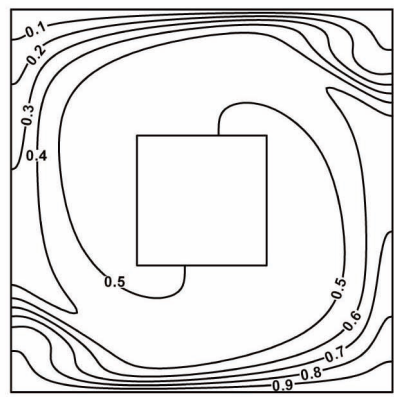

(b-1) Isotherms for $\operatorname{Pr}=0.7$

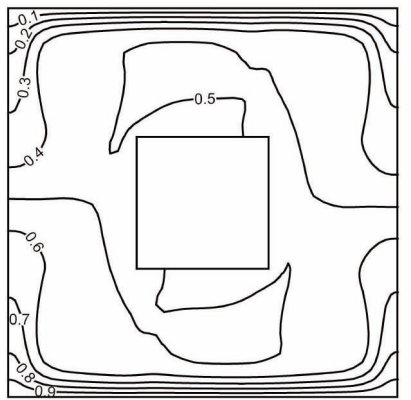

(c-1) Isotherms for $\operatorname{Pr}=7$

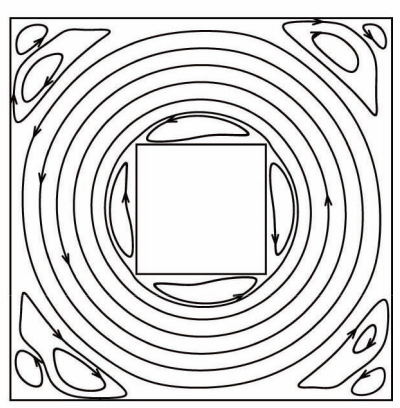

(a-2) Streamlines for $\operatorname{Pr}=0.01$

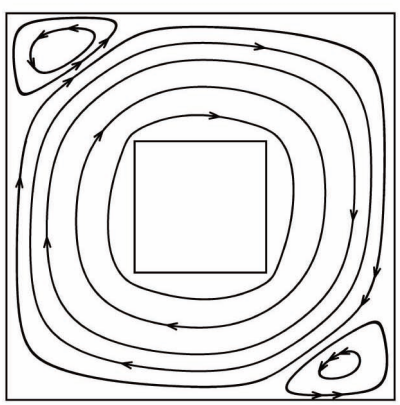

(b-2) Streamlines for $\operatorname{Pr}=0.7$

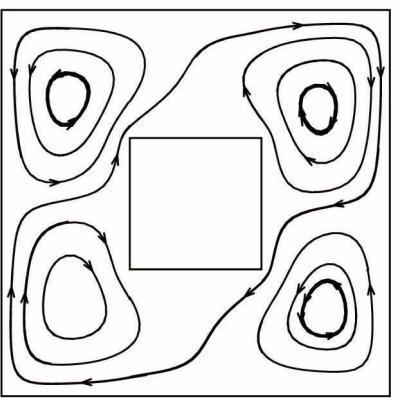

(c-2) Streamlines for $\operatorname{Pr}=7$
Fig. 12. Distribution of the Isotherms and Streamlines at $\mathrm{Ra}=10^{6}$.

that the pattern is similar to those obtained for $\mathrm{Ra}=10^{5}$. The difference between Fig. 12(a) and Fig. 8(a) is that the thermal gradients near the isothermal boundary for $\mathrm{Ra}=10^{6}$ become denser and the secondary vortices increase their strength at the corners. Fig. 12(c) shows the long-time averaged isotherms and streamlines for $\mathrm{Pr}=7$. As shown in Fig. 11(k), the duration for clockwise and counterclockwise circulations are not the same and the long-time averaged solution does not satisfy the $\mathrm{x}$ - and $\mathrm{y}$-symmetry but fulfills the diagonal symmetry in the same manner as the other two Pr cases for the given Ra.

\section{Time- and surface-averaged Nusselt numbers}

Fig. 13 shows a time- and surface-averaged Nusselt number at the hot bottom wall as a function of the Rayleigh

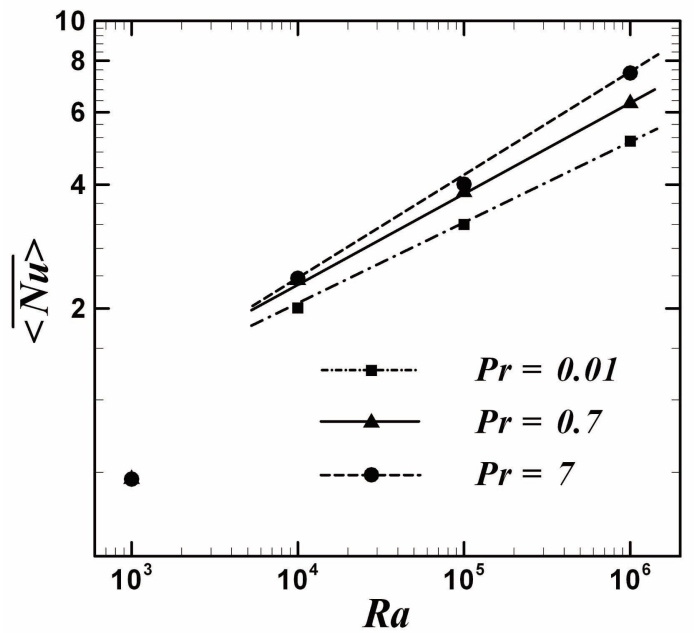

Fig. 13. Time- and Surface-averaged Nusselt Number at the Hot and Cold walls as a Function of the Rayleigh Number for Different Prandtl Numbers.

number. The time- and surface-averaged Nusselt number shows the same value for every Prandtl number case for $\mathrm{Ra}=10^{3}$ at which an onset of convective flow does not occur so that the thermal states are dominantly governed by the heat conduction. As the Ra increases, the buoyant force becomes larger than the critical Rayleigh number and then the convective fluid flow is activated. For the convection regime, the larger the $\mathrm{Pr}$ is, the higher the averaged heat transfer rate. The values shown are logarithmically linear to the Rayleigh number in the convection regime.

\section{CONCLUSIONS}

We investigated thermal convection in a horizontal enclosure of a fluid layer with an adiabatic obstacle located in the middle for different viscous properties, using an accurate and efficient Chebyshev spectral collocation approach. A multi-domain methodology was employed to address the geometric complexity introduced by the adiabatic body in the middle of an enclosure.

When the Rayleigh number is moderately small $\left(\mathrm{Ra}=10^{4}\right)$, the flows go to the stable steady state regardless of their Prandtl numbers. However, the time needed to break down the initial $\mathrm{x}$ - and $\mathrm{y}$-symmetry is affected by the Prandtl number. As the Prandtl number increases, the breakdown occurs earlier.

As the Rayleigh number increases, the stability of the convective flow becomes dependent on the Prandtl number. The case of $\mathrm{Pr}=0.01$ shows the roll cell and how the secondary vortices change their shape periodically but do not change their directions. The cases of higher Prandtl number $(\mathrm{Pr}=0.7$ and 7$)$ do not show such oscillatory behavior untill $\mathrm{Ra}=10^{5}$. However, if the $\mathrm{Ra}$ increases to 
$10^{6}$, the case of high Prandtl number $(\mathrm{Pr}=7)$ also shows an oscillatory thermal fluidic behavior in which the circulating direction repeatedly changes from clockwise and counterclockwise, but in the case of a moderate Prandtl number $(\operatorname{Pr}=0.7)$ not much oscillatory behavior occurs, that is, there is a specific band of Pr number at which the flow prior to the transition region is stable. This result suggests a guideline to understand a heat transport system using non-unity fluidic medium.

The time- and surface-averaged Nusselt number at the hot bottom wall is logarithmically linear as the Rayleigh number increases in a convective regime. For further general physical consistency, a study for a wide range of Rayleigh numbers and Prandtl numbers should be conducted in the near future. It will also be necessary to carry out threedimensional calculation for a further range of Rayleigh numbers, such as for a turbulent regime.

\section{NOMENCLATURE}

AD = advection terms in the Navier-Stokes equation

$g \quad=$ gravity

$L \quad=$ length of the enclosure

n $=$ vector normal to the surface

NL = nonlinear and buoyancy terms in Navier-Stokes equation

$N u=$ local Nusselt number $(=\partial \theta / \partial \mathbf{n})$

$\overline{N u}=$ surface-averaged Nusselt number

$\langle\mathrm{Nu}\rangle=$ time- and surface-averaged Nusselt number

$p \quad=$ dimensionless pressure

pr $\quad=$ Prandtl number $(=v / \alpha)$

$T=$ dimensional temperature

$t \quad=$ dimensionless time

$\mathbf{u}=$ dimensionless velocity

$\mathbf{x}=$ dimensionless coordinate vector

$\alpha \quad=$ thermal diffusivity

$\beta=$ thermal expansion coefficient

$v \quad=$ kinematic viscosity of fluid

$\theta=$ dimensionless temperature

$\rho=$ density

\section{Subscripts}

* $\quad=$ intermediate step

body $=$ body

$c \quad=$ cold

$h=$ hot

$n \quad=$ present step

$p \quad=$ period

wall $=$ wall

\section{Superscripts}

$=$ dimensional variable

\section{REFERENCES}

[1] P.G. Drazin and W.H. Reid, Hydrodynamic stability, Cambridge University Press, UK, (1981)
[2] F.B. Lipps, Numerical simulation of three-dimensional bénard convection in air, Journal of Fluid Mechanics, 75 pp.113-148 (1976)

[3] S. Balachandar, M.R. Maxey and L. Sirovich, Numerical simulation of high Rayleigh number convection, Journal of Scientific Computing, 4, pp.219-236 (1988)

[ 4 ] D.P. McKenzie, J.M. Roberts and N.O. Weiss, Convection in the earth's mantle: towards a numerical simulation, Journal of Fluid Mechanics, 62, pp.465-538 (1974)

[5] A. Nakano, H. Ozoe and S.W. Churchill, Numerical computation of natural convection for a low-Prandtl-number fluid in a shallow rectangular region heated from below, Chemical Engineering Journal, 71, pp.175-182 (1998)

[6] T. Pesso and S. Piva, Laminar natural convection in a square cavity: Low Prandtl numbers and large density differences, International Journal of Heat and Mass Transfer, 52, pp.10361043 (2009)

[ 7 ] R. Verizicco and R. Camussi, Prandtl number effects in convective turbulence, Journal of Fluid Mechanics, 383, pp.55-73 (1999)

[ 8 ] R. Simitev and F.H. Busse, Prandtl-number dependence of convection-driven dynamos in rotating spherical fluid shells, Journal of Fluid Mechanics, 532, pp.365-388 (2005)

[9] S.K. Choi and S.O. Kim, Treatment of turbulent heat fluxes with the elliptic-blending second-moment closure for turbulent natural convection flows, International Journal of Heat and Mass Transfer, 51, pp.2377-2388 (2008)

[10] B.J.Yun et al. Construction Report of Separate Effect Test Facility for Passive Auxiliary Feedwater System (PASCAL), KAERI/TR-4085/2010 (2010)

[11] J. Kim et al. Analysis of a Molten Pool Natural Convection in the APR1400 RPV at a Severe Accident, Transaction of the Korean Nuclear Society Spring Meeting, Jeju, Korea, May 26-27 (2005)

[12] H. Lee and G.C. Park, Turbulent Natural Convection in a Hemispherical Geometry Containing Internal Heat Sources, Journal of Korean Nuclear Society, 30, pp.496-506 (1998)

[13] G de, Vahl Davis, Natural Convection of Air in a Square Cavity: A Bench Mark Numerical Solution, International Journal of Numerical Methods in Fluids, 3, pp.249-264 (1983)

[14] J.M. House, C. Beckermann and T.F. Smith, Effect of a centered conducting body on natural convection heat transfer in an enclosure, Numerical Heat Transfer, Part A, 18, 213225 (1990)

[15] Q.H. Deng, G.F. Tang, Numerical visualization of mass and heat transport for conjugate natural convection/heat conduction by streamline and heatline, International Journal of Heat and Mass Transfer, 45, pp.2373-2385 (2002)

[16] A. Mezrhab, H. Bouali, H. Amaoui, M.Bouzidi, Computation of combined natural-convection and radiation heat-transfer in a cavity having a square body at its center, Applied Energy, 83, pp.1004-1023 (2006)

[17] H.S. Yoon, M.Y. Ha, B.S. Kim and D.H. Yu, Effect of the position of a circular cylinder in a square enclosure on natural convection at Rayleigh number of $10^{7}$, Physics of Fluids, 21, 047101-1 - 047101-11 (2009)

[18] H.K. Jeong, H.S. Yoon, M.Y. Ha and M. Tsutahara, An immersed boundary-thermal lattice Boltzmann method using an equilibrium internal energy dendisy approach for the simulation of flows with heat transfer, Journal of 
Computational Physics, 229, pp.2526-2543 (2010)

[19] M.Y. Ha, H.S. Yoon, K.S. Yoon, S. Balachandar, I. Kim, J.R. Lee and H.H. Chun, Two-Dimensional and Unsteady Natural Convection in a Horizontal Enclosure with a Square Body, Numerical Heat Transfer, Part A, 41, pp.183-210 (2002)

[20] C.L. Streett and M.G. Macaraeg, Spectral Multi-Domain for Large-Scale Fluid Dynamic Simulations, Applied Numerical Mathematics, 6, 123-139 (1989)

[21] C. Canuto, M.Y. Hussaini, A. Quarteroni, and T.A. Zang, Spectral Methods in Fluid Dynamics, Springer Verlag, (1988)

[22] S.J. Parker, Stability and vortex shedding of bluff body arrays, PhD Thesis, University of Illinois, Urbana, IL, (2002)

[23] P. Le Quere, Accurate solution to the square thermally driven cavity at high Rayleigh number, Computers \& Fluids, 20, pp.29 (1991)

[24] H.S. Yoon, K.V. Sharp, D.F. Hill, R.J. Adrain, S. Balachandar, M.Y. Ha and K. Kar, Integrated Experimental and Computational Approach of Flow in a Stirred Tank, Chemical Engineering Science, 56, 3714-3728 (2001)

[25] D.H. Lee, M.Y. Ha, S. Balachandar and S.S. Lee, Numerical Simulations of Flow and heat transfer Past a Circular Cylinder with a periodic array of Fins, Physics of Fluids, $16,1273-1286$ (2004)

[26] R.E. Kelly and D. Pal, Thermal convection with spatially periodic boundary conditions: resonant wavelength excitation,
Journal of Fluid Mechanics, 86, 433-456 (1978)

[27] J.R. Lee, M.Y. Ha, S. Balachandar, H.S. Yoon and S.S. Lee, Natural convection in a horizontal layer of fluid with a periodic array of square cylinders in the interior, Physics of Fluids, 16, pp.1097-1117 (2004)

[28] D. Puigjaner, J. Herrero, C. Simó and F. Giralt, Bifurcation analysis of steady Rayleigh-Bénard convection in a cubical cavity with conducting sidewalls, Journal of Fluid Mechanics, 598 (2008) 393-427

[29] H. Bertin and H. Ozoe, Numerical study of two-dimensional natural convection in a horizontal fluid layer heated from below, by finite-element method: influence of Prandtl number, International Journal of Heat and Mass Transfer, 29, 439-449 (1986)

[30] M.Y. Ha, I.K. Kim, H.S. Yoon, and S. Lee, Unsteady fluid flow and temperature fields in a horizontal enclosure with an adiabatic body, Physics of Fluids 14, pp.3189-3202 (2002)

[31] R.M. Clever and F.H. Busse, Convection at very low Prandtl number, Physics of fluids, 2, pp.334-339 (1990)

[32] H. Ozoe and T. Hara, Numerical analysis for oscillatory natural convection of low Prandtl number fluid heated from below, Numerical Heat Transfer Part A, 27, pp.307-317 (1995)

[33] S. Arcidiacono, I. Di Piazza, M. Ciofalo, Low-Prandtl number natural convection in volumetrically heated rectangular enclosures II Square cavity, $\mathrm{AR}=1$, International Journal of Heat and Mass Transfer, 44, pp.537-550 (2001) 